\title{
A ultra-sonografia diaǵnóstica (modo B) na avaliação pós-operatória das cirurgias vítreo-retinianas
}

\author{
B-scan ultrasound in the postoperative evaluation of vitreoretinal surgery
}

\author{
Zélia Maria da Silva Corrêa ${ }^{1}$ \\ Ítalo Mundialino Marcon ${ }^{2}$
}

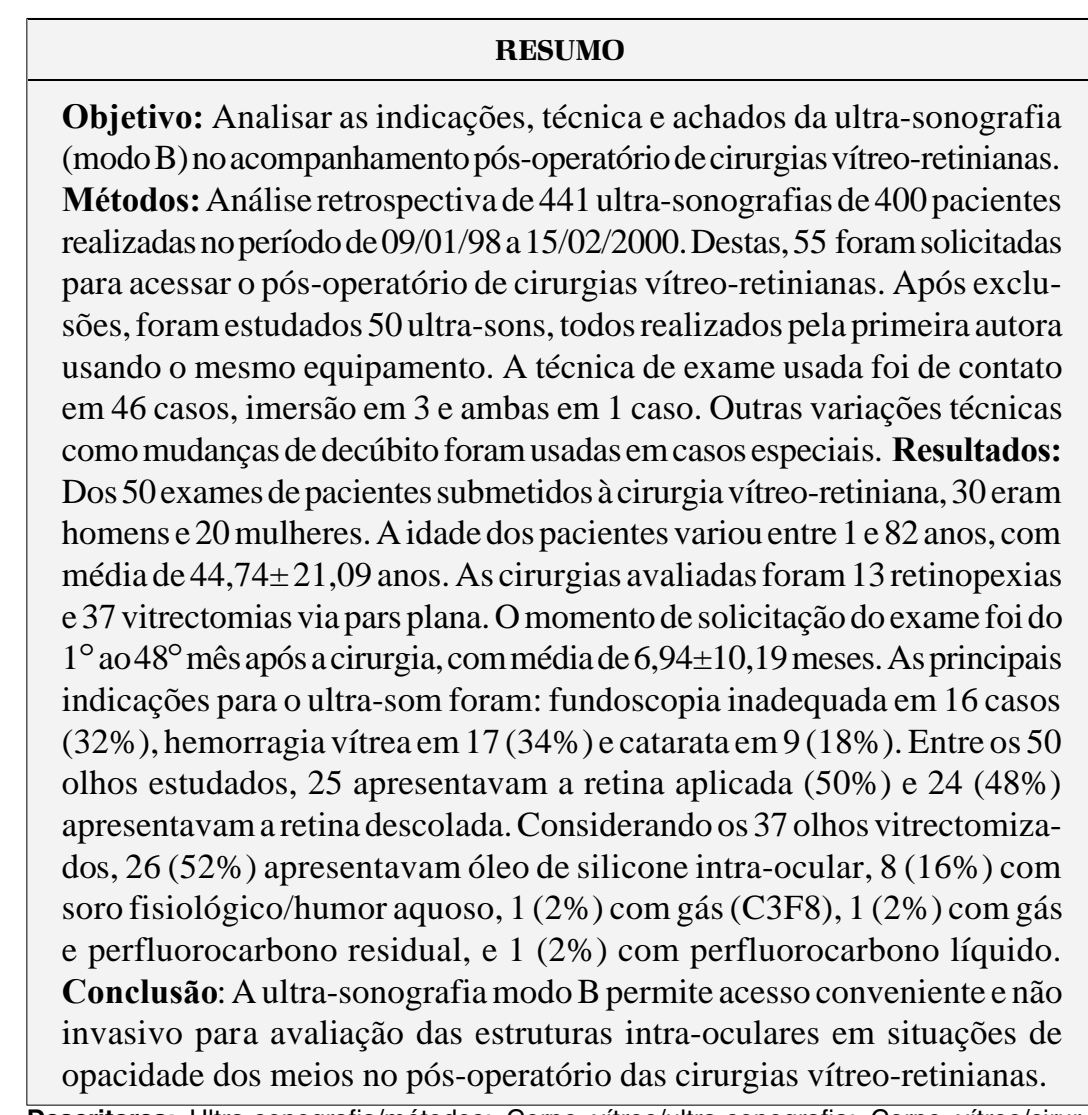

Descritores: Ultra-sonografia/métodos; Corpo vítreo/ultra-sonografia; Corpo vítreo/cirurgia; Período pós-operatório; Estudos de avaliação
${ }^{1}$ Pós-graduanda, nível doutorado pela Universidade Federal de São Paulo - UNIFESP. Instituto de Olhos Ítalo M. Marcon e Serviço de Oftalmologia da Santa Casa de Porto Alegre (ISCMPA), Porto Alegre, RS. ${ }^{2}$ Doutor em Oftalmologia pela Universidade Federal de São Paulo - UNIFESP. Fundação Faculdade Federal de Ciências Médicas de Porto Alegre (FFFCMPA), Porto Alegre, RS.

Endereço para correspondência: Avenida Nilo Peçanha, 2421 - Porto Alegre (RS) CEP 91330-001.

E-mail: zmcorrea@zaz.com.br

Recebido para publicação em 18.01.2001 Aceito para publicação em 14.08.2002

Nota Editorial: Pela análise deste trabalho e por sua anuência na divulgação desta nota, agradecemos à Dra. Vera Regina Cardoso Castanheira.

\section{INTRODUÇ̃̃̃O}

A ultra-sonografia diagnóstica em oftalmologia, desenvolvida no final dos anos 50 e início da década de 60, permanece um método essencial de exploração e diagnóstico de patologias do globo ocular e órbita ${ }^{(1)}$. Suas indicações vão desde as opacidades dos meios causadas por leucoma de córnea, catarata, membrana ciclítica até hemorragia vítrea, endoftalmite, trauma e tumor ocular ${ }^{(2-3)}$. Este acessório diagnóstico tem sido cada vez mais utilizado na prática em casos de pós-operatórios de cirurgia vítreoretiniana quando há impossibilidade de visualizar o fundo ou há necessidade de confirmação de certas complicações cirúrgicas como hemorragia vítrea, hemorragia intra-coroidal localizada, descolamento de retina recorrente, introflexão escleral muito apertada ou até hipotonia ocular ${ }^{(4-6)}$. 
O avanço dos equipamentos ultra-sonográficos em relação à resolução das imagens, associadas à técnica de imersão e cinética, melhoraram as informações obtidas no pós-operatório da cirurgia vítreo-retiniana. Além disso, o ultra-som é inócuo, simples de executar, de custo relativamente baixo e possibilita avaliar as dimensões e a constituição intrínseca das lesões, levando esse método de exame a tornar-se indispensável no arsenal diagnóstico da oftalmologia ${ }^{(1)}$. Deve-se ressaltar que a existência de óleo de silicone ou gás intra-ocular ainda representa um obstáculo na interpretação dos achados e na obtenção de imagens adequadas ${ }^{(1,5)}$, exigindo do examinador experiência para associar variadas técnicas e manobras bem como conhecimento amplo das patologias oculares.

Este estudo tem como objetivo analisar as indicações, modo de execução e exemplificar os achados da ultra-sonografia (modo B) no acompanhamento pós-operatório de pacientes submetidos à cirurgia vítreo-retiniana.

\section{MÉTODOS}

Foram avaliados retrospectivamente todos os exames ecográficos realizados no período de 09/01/98 a 15/02/2000 no Departamento de Oftalmologia da Santa Casa de Porto Alegre (ISCMPA) e no Instituto de Olhos Ítalo M. Marcon, ambos em Porto Alegre, RS, num total de 441 exames de 400 pacientes. Destes, 55 exames foram solicitados com o objetivo de acessar o pós-operatório de cirurgias vítreo-retinianas. Foram excluídos os casos cujos dados pessoais, ou motivo para solicitação do exame estavam incompletos. Após exclusões, 50 exames foram revisados, considerando-se apenas o primeiro exame realizado em cada paciente, quanto ao motivo da solicitação, idade e sexo dos pacientes, os achados ecográficos destacando a posição da retina, presença de gás ou silicone intraocular, alterações vítreas ou da coróide e as modificações da técnica usual para melhor definição das imagens.

Todos os exames solicitados foram realizados pela primeira autora com o aparelho Advent A/B System ${ }^{\circledR}$, Mentor O\&O Inc, Norwell, MA; cuja sonda possui intensidade variável entre 7,5 e $15 \mathrm{MHz}$, mas todos os exames aqui citados utilizaram a sonda de $15 \mathrm{MHz}$. A técnica de exame usada foi de contato sobre a pálpebra (transpalpebral) com uso de gel para ecografia $\left(\right.$ Sonics $\left.^{\circledR}\right)$ na sonda ou em contato direto com globo ocular (imersão), mediante colocação de blefarostato e usando um dedo de luva (látex) adaptado à sonda e preenchido com soro fisiológico $0,9 \%$. Ambas as técnicas foram realizadas com o paciente em decúbito dorsal horizontal após anestesia tópica com proparacaína colírio. Em casos especiais foram realizadas mudanças de decúbito (supina e lateral) associadas a movimentos oculares para a obtenção de imagens cinéticas do vítreo e retina, com menor quantidade de artefatos.

Os dados obtidos receberam tratamento estatístico com cálculo de freqüências usando o programa SPSS, versão 6.1, Chicago, Illinois, USA.

\section{RESULTADOS}

Dos 50 exames estudados de pacientes submetidos à cirurgia vítreo-retiniana, 30 eram do sexo masculino e 20 do feminino. A idade dos pacientes variou entre 1 e 82 anos, com média de 44,74 anos e desvio padrão de 21,09. O estudo ultra-sonográfico foi realizado na mesma proporção em olhos direitos $(n=25)$ e esquerdos $(n=25)$. As cirurgias avaliadas foram 13 retinopexias e 37 vitrectomias via pars plana. O momento de solicitação do exame foi do $1^{\circ}$ ao $48^{\circ}$ mês após a cirurgia vítreoretiniana, com média de 6,94 meses e desvio padrão de 10,19.

As indicações para o estudo foram: fundoscopia inadequada em 16 casos (32\%), turvação inflamatória do vítreo em 5 casos (10\%), hemorragia vítrea em 17 casos (34\%), catarata em 9 casos (18\%), descompensação do endotélio corneano em 2 casos (4\%) e baixa da acuidade visual a esclarecer em 1 caso (2\%) (Tabela 1). Foram consideradas causas de fundoscopia inadequada $(n=16)$ : edema corneano transitório $(n=9)$, midríase insatisfatória $(n=4)$ e membrana pupilar ciclítica $(n=3)$. Considerando os 50 casos, somente um deles oferecia condições para realizar oftalmoscopia indireta. Nesse caso, cuja indicação do exame foi baixa da acuidade visual a esclarecer, o ultrasom possibilitou o diagnóstico de espessamento da coróide difuso que não havia sido percebido à oftalmoscopia indireta apesar da pressão intra-ocular não ter se apresentado inferior a $8 \mathrm{mmHg}$ durante o pós-operatório.

Entre os 50 olhos estudados, 26 apresentavam óleo de silicone intra-ocular (52\%), 1 (2\%) apresentava gás (C3F8), 1 (2\%) apresentava resíduo de perfluoro-carbono líquido associado à hemorragia vítrea e intra-coroídea e outro caso (2\%), gás e resíduo de perfluorocarbono líquido (Tabela 2). Nesse último, foi impossível distinguir as condições da retina apesar das diferentes técnicas (imersão e mudança de decúbito) usadas para minimizar o efeito dos artefatos. Cerca de 20 dias depois o paciente foi reavaliado e só após a reabsorção do gás foi possível observar, ao ultra-som modo $\mathrm{B}$, a retina descolada devido à proliferação vítreo-retiniana e sub-retiniana e a presença de perfluorocarbono líquido sub-retiniano. Em 3 casos houve necessidade de técnica de imersão para melhorar a

Tabela 1. Freqüência das indicações de ecografia no pós-operatório de cirurgia vítreo-retiniana

\begin{tabular}{|c|c|c|}
\hline Indicações & № casos & $\%$ \\
\hline Fundoscopia inadequada* & 16 & 32 \\
\hline Hemorragia vítrea & 17 & 34 \\
\hline Catarata & 9 & 18 \\
\hline Turvação inflamatória do vítreo\# & 5 & 10 \\
\hline Leucoma corneano & 2 & 4 \\
\hline Baixa da acuidade visual & 1 & 2 \\
\hline Total & 50 & 100 \\
\hline \multicolumn{3}{|c|}{$\begin{array}{l}\text { *Consideradas causas de fundoscopia inadequada }(n=16) \text { : edema de córnea } \\
(n=9) \text {, midríase pupilar insatisfatória }(n=4) \text { e membrana pupilar }(n=3) \text {. } \\
\text { "Foram agrupados como "Turvação inflamatória do vítreo" os casos de turvação } \\
\text { vítrea inespecífica por debris e condensações }(n=3) \text { e aqueles com aspecto } \\
\text { sugestivo de vitreíte }(n=2) \text {. }\end{array}$} \\
\hline
\end{tabular}




\begin{tabular}{|c|c|c|c|}
\hline \multirow[t]{2}{*}{ Achados ecográficos } & \multicolumn{3}{|c|}{ Status da retina $(n=50)$} \\
\hline & $\begin{array}{l}\text { Aplicada } \\
(n=25)\end{array}$ & $\begin{array}{c}\text { Descolada } \\
(n=24)\end{array}$ & $\begin{array}{l}\text { Inconclu- } \\
\text { sivo }(n=1)\end{array}$ \\
\hline Hemorragia de coróide* & $3(6 \%)$ & - & - \\
\hline Espessamento da coróide & $4(8 \%)$ & $1(2 \%)$ & - \\
\hline Proliferação vítreo-retiniana & - & $13(26 \%)$ & - \\
\hline Tração vítrea & - & $9(18 \%)$ & - \\
\hline Defeito retiniano" & - & $1(2 \%)$ & - \\
\hline Conteúdo intra-ocular no & segmento & posterior & \\
\hline Humor vítreo & $3(6 \%)$ & $10(20 \%)$ & - \\
\hline Humor aquoso & $4 \quad(8 \%)$ & $4(8 \%)$ & - \\
\hline Silicone intra-ocular & $16(32 \%)$ & $10(20 \%)$ & - \\
\hline Gás intra-ocular & $1(2 \%)$ & - & - \\
\hline Gás + perfluor ${ }^{\circ}$ & - & - & $1(2 \%)$ \\
\hline Perfluorcarbono residual & $1(2 \%)$ & - & - \\
\hline \multicolumn{4}{|c|}{$\begin{array}{l}\text { A freqüência dos achados foi apresentada seguida da porcentagem do total de } \\
\text { pacientes estudados ( } n=50) \text { * }{ }^{*} \text { termo hemorragia de coróide foi empregado } \\
\text { para os casos de hemorragia intra-coroidal localizada. " } O \text { termo "defeito } \\
\text { retiniano" foi usado para o caso onde o rasgo da retina não estava bloqueado. } \\
\text { " Gás+perfluor corresponde à presença de gás intra-ocular e perfluorcabono } \\
\text { líquido residual e foi o único caso onde não foi possível obter imagens ultra- } \\
\text { sonográficas conclusivas e acessar o status da retina }\end{array}$} \\
\hline
\end{tabular}

captação dos ecos e no restante $(n=46)$, foi usada a técnica de contato transpalpebral. Os casos onde foi usada a técnica de imersão, foram situações onde o olho examinado era pseudofácico ou havia silicone na câmara anterior. Esta técnica permitiu observar detalhes do conteúdo intra-ocular que seria impossível de outra forma. A mudança de decúbito foi usada em 18 casos, sempre após vitrectomia via pars plana, quando havia gás, silicone, perfluorocarbono intra-ocular ou associação destes, e casos especiais onde o paciente não conseguia se manter no decúbito dorsal devido à patologia respiratória $(n=1)$ e ortopédica $(n=1)$.

Vinte e cinco olhos apresentavam a retina aplicada $(50 \%)$, sendo que $3(6 \%)$ apresentavam hemorragia intra-coroidal localizada, e 4 (8\%) apresentavam espessamento da coróide e hipotonia secundária. Vinte e quatro olhos (48\%) apresentavam a retina descolada, sendo que 13 (26\%) apresentavam proliferação vítreo-retiniana, $9(18 \%)$ apresentavam tração vítrea, $2(4 \%)$ apresentavam defeito retiniano não bloqueado e 1 apresentou espessamento da coróide e globo ocular diminuído de tamanho (pré-phthisis).

\section{DISCUSSÃO}

Alguns conceitos fundamentais devem ser considerados na análise de imagens ultra-sonográficas como: a dinâmica do exame, a escala em tons de cinza, a intensidade dos ecos, a perpendicularidade na obtenção das imagens e a imagem tri-dimensional que o examinador deve criar mentalmente para entender o desenrolar do estudo(7). Esta última é, sem dúvida, a mais difícil e demorada para dominar ${ }^{(1)}$.
A ecografia modo B tem sido extensivamente utilizada na avaliação pré-operatória das cirurgias vítreo-retinianas, especialmente vitrectomias, e seus achados têm se mostrado de alta confiabilidade (entre $77,1 \%$ a $92,2 \%$ dependendo da patologia) ${ }^{(8)}$. Entretanto existem poucos relatos na literatura nacional acerca dos achados ecográficos pós-cirurgia vítreo-retiniana, limitados aos livros-texto especializados ${ }^{(5-6,9)}$.

A cerclagem ou "buckle" escleral aparece como uma estrutura hipoecogênica cujo tamanho e formato dependem do tipo de implante colocado. Geralmente percebe-se uma sombra atrás do buckle devido à atenuação do som ao atravessar esta estrutura. (Figura 1). Olhos com hemorragia vítrea podem ser avaliados em mais detalhes pela ecografia ${ }^{(3,9)}$. Detalhes estruturais podem ser mais bem acessados com a diminuição do ganho ("gain") do aparelho para reduzir os ecos vítreos ${ }^{(7)}$. Isto facilita a visualização de descolamentos de retina ou traves fibrovasculares obscurecidas pela ecogenicidade observada em hemorragias e coágulos.

A presença de gases muitas vezes inviabiliza o exame devido a total reflexão dos ecos na interface líquido-ar. A mudança da posição da cabeça ou até de decúbito do paciente, associada a movimentos oculares evidenciam detalhes essenciais, da anatomia e cinética do vítreo e retina, para o diagnóstico ecográfico. Além disso, cortes ecográficos diferentes podem proporcionar exames interpretáveis ${ }^{(5,10-11)}$.

O silicone intra-ocular, se for uma bolha única, causa somente um aumento aparente do diâmetro axial do olho devido à menor velocidade do som neste meio (normal $=1584 \mathrm{~m} / \mathrm{s}$, silicone $=980 \mathrm{~m} / \mathrm{s})^{(5,10)}$. Tal situação pode ser resolvida em aparelhos onde o examinador pode mudar a velocidade padrão do som ${ }^{(1)}$ ou "parcialmente contornada" aumentado o comprimento a ser estudado, no caso do aparelho usado neste estudo, mudou-se o alcance de $35 \mathrm{~mm}$ para $50 \mathrm{~mm}$ (Figura 2).

Kumar e colaboradores estudaram a influência da mudança de decúbito no resultado da ecografia em olhos com óleo de silicone ${ }^{(10)}$. Os autores perceberam que o exame em pronação facilita a avaliação da retina por eliminar os 2 ecos quase consecutivos dados pela interface do silicone e a esclera. Este fato mudava a eficácia diagnóstica da ecografia modo B de $61,8 \%$ (posição supina) para 96,7\% (associação das posições supina e pronação) $)^{(10)}$. No presente estudo foram usados os decúbitos supino e lateral para a realização das ultra-sonografias. Apesar disto estas imagens podem ser enganadoras e requerem experiência em sua interpretação. Caso o silicone esteja parcialmente emulsificado, torna-se um pouco mais confuso de ser interpretado, restos de silicone no olho aparecem como asteróides (Figura 2). As relações entre as estruturas intra-oculares podem ser analisadas de maneira dinâmica o que facilita a interpretação das imagens quando há líquidos ou gases intra-oculares.

Hasenfratz e colaboradores estudaram olhos com resíduos de perfluorocarbono com ecografia padrão A e ecografia diagnóstica modo $\mathrm{B}^{(12)}$. Ambas modalidades ecográficas revelaram a diminuição da velocidade do ultra-som quando atravessava 


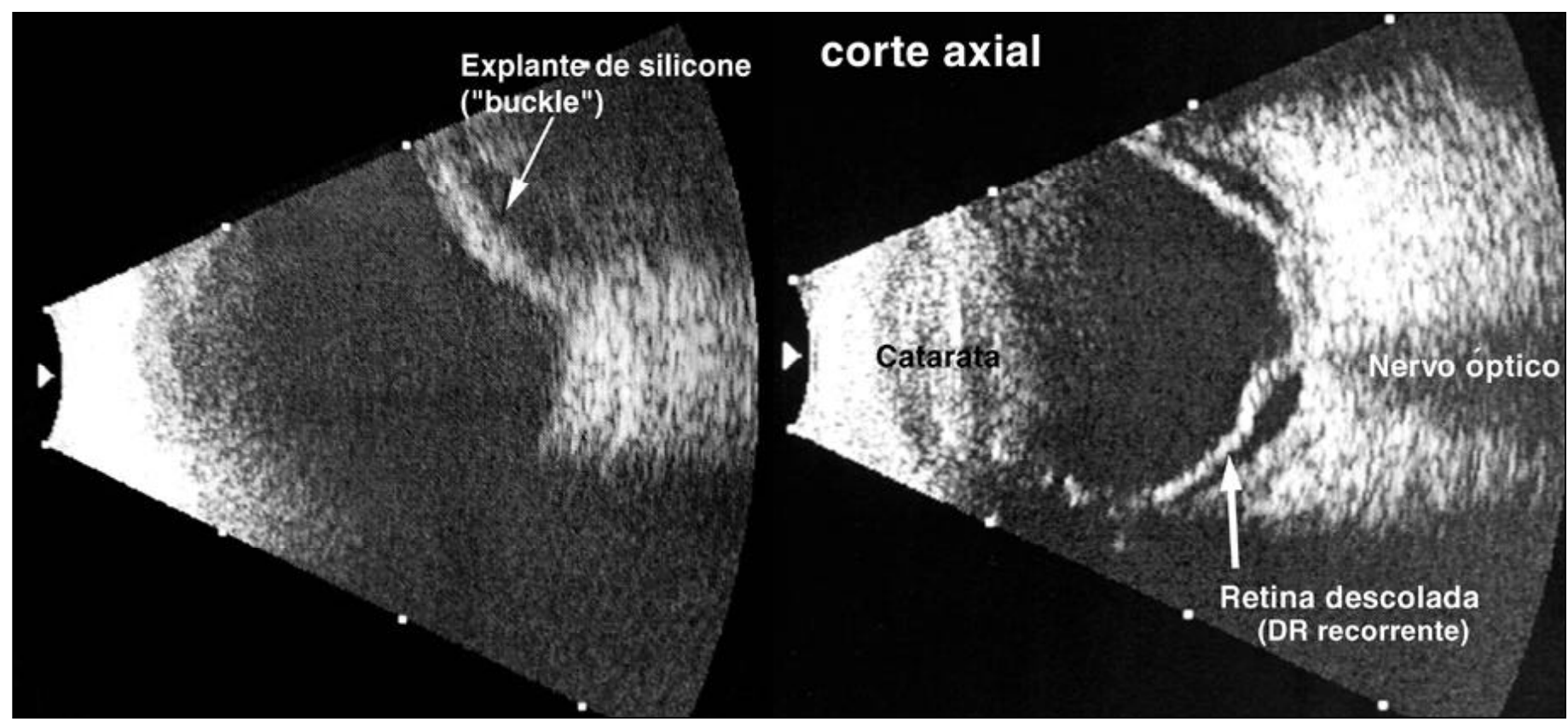

Figura 1 - Ultra-sonografias modo B no pós-operatório de introflexão escleral. A imagem esquerda revela retina aplicada após procedimento de retinopexia com introflexão escleral, observe a endentação escleral provocada pelo explante de silicone e a sombra acústica projetada pelo mesmo. A imagem direita revela descolamento de retina recorrente (indicado pela flecha) com inserção na papila

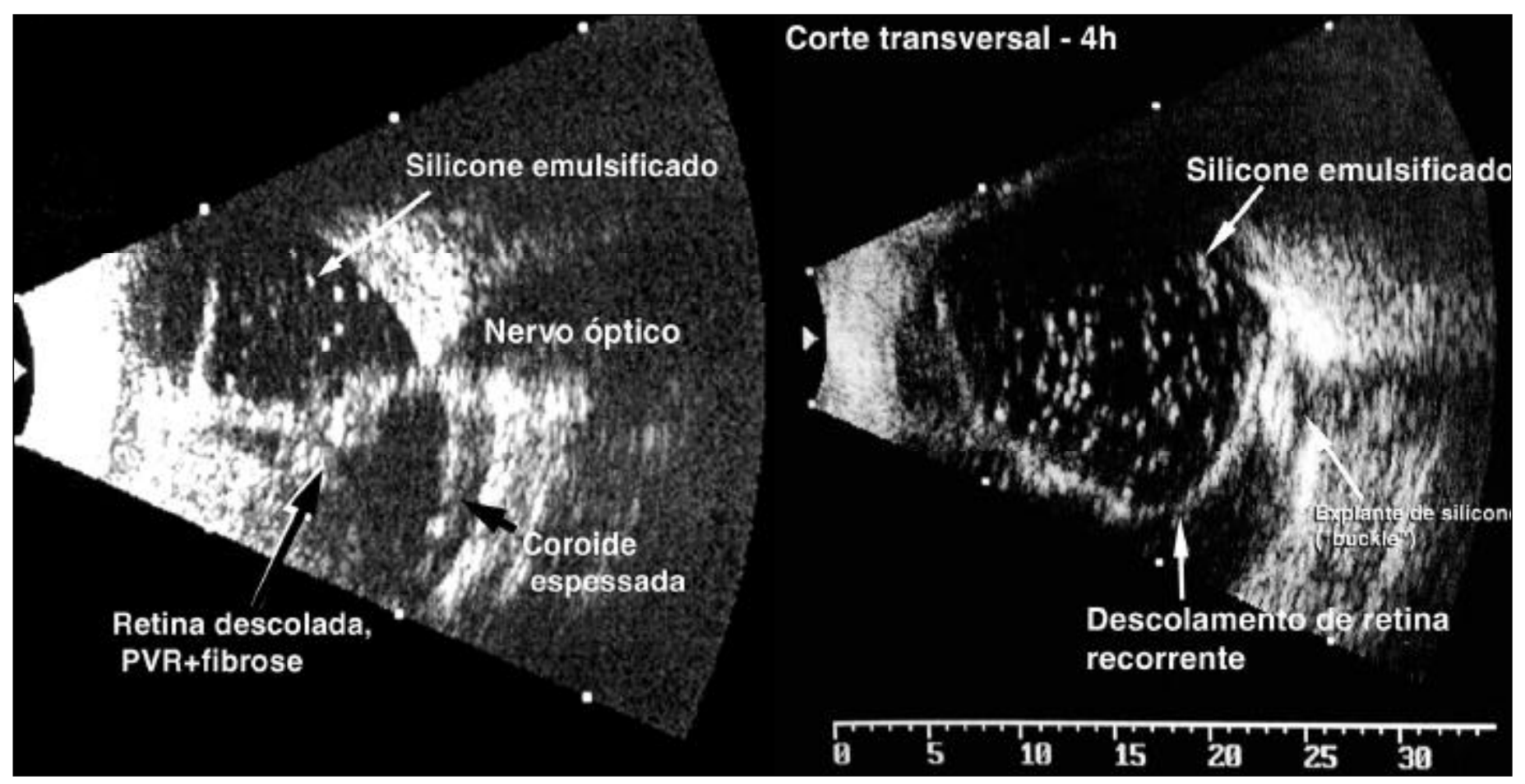

Figura 2 - Ultra-sonografia modo B no pós-operatório de vitrectomia via pars plana e complicações após tamponamento com óleo de silicone. Nota-se $o$ aspecto característico de silicone emulsificado intra-ocular em ambas as imagens. A imagem esquerda revela corte axial de globo ocular com retina descolada, PVR intenso, fibrose e migração do silicone para o espaço sub-retiniano. A imagem direita ilustra descolamento de retina não regmatogênico recorrente e silicone emulsificado na cavidade vítrea. (Legendas: PVR = proliferação vítreo-retiniana)

o perfluorcarbono. Entretanto a diferenciação mais segura, com outras alterações vítreo-retinianas, foi obtida com a ecografia padrão $\mathrm{A}^{(12)}$.
As imagens obtidas em olhos com perfluorocarbono revelam sombra acústica no local deste fluido pesado e a diminuição da velocidade do som como descrito por Hasenfratz. A 


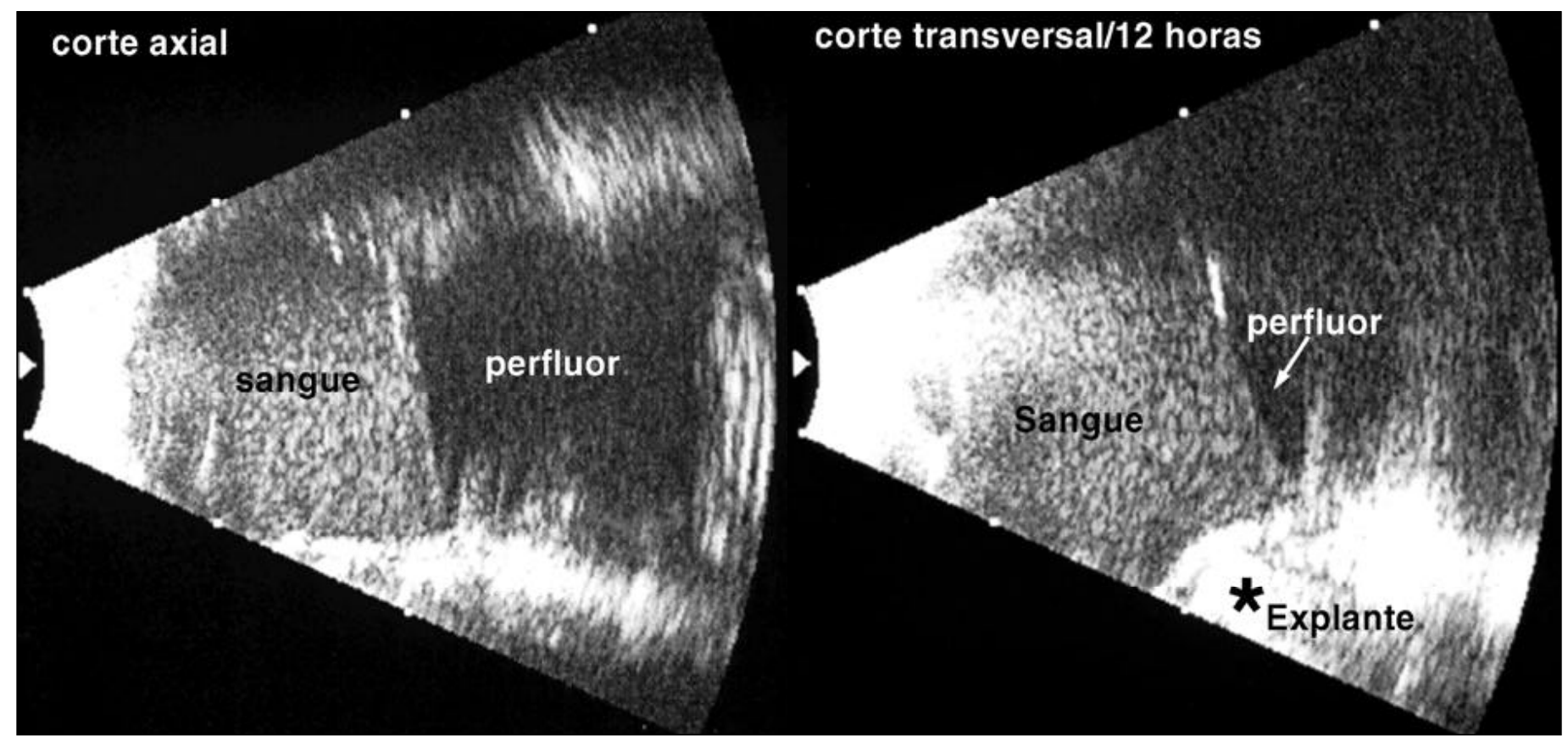

Figura 3 - Ultra-sonografias modo $B$ de paciente submetido à vitrectomia via pars plana e introflexão escleral há 1 mês com hemorrgia vítrea e da coróide no final do procedimento e tamponamento temporário com perfluorocarbono líquido. A imagem esquerda revela sangue no segmento posterior e sombra acústica causada pela presença do perfluorocarbono. A imagem direita realizada deslocando a sonda superior, mantendo o globo ocular na posição primária do olhar, possibilita visualizar o explante de silicone. (sangue = hemorragia vítrea, perfluor = perfluorocarbono líquido)

mudança de posição da sonda pareceu ajudar a obtenção das imagens (Figura 3). As maiores dificuldades ocorrem quando existe uma mistura entre gases e fluidos usados na cirurgia vítreo-retiniana como, por exemplo, gás e perfluorocarbono, gás somente e óleo de silicone. Sendo que a primeira combinação impossibilitou a captação de imagens interpretáveis ao ultra-som e as demais foram minimizadas por técnicas de exame descritas nesse estudo.

\section{CONCLUSÃO}

Os achados desse estudo mostram que a ultra-sonografia modo B oferece um acesso conveniente e não-invasivo para avaliação das estruturas intra-oculares em situações de opacidade dos meios no pós-operatório das cirurgias vítreo-retinianas, mantendo, apesar da evolução tecnológica dos aparelhos, limitações nos exames de olhos que contém gás ou silicone intra-ocular. Nestas situações a boa experiência do profissional, a associação de técnicas como a imersão ou a ecografia dinâmica e as mudanças de decúbito, retirando os ecos anômalos da frente das estruturas de interesse, colaboram para minimizar as dificuldades e aumentam a sensibilidade diagnóstica desse método tão útil para nossa especialidade.

\section{ABSTRACT}

Purpose: To evaluate the indications, techniques and findings of B-scan ultrasound after vitreoretinal surgery.
Methods: Retrospective study of $441 \mathrm{~B}$-scan ultrasounds of 400 patients between 01/09/98 and 02/15/00. Of these, 55 examinations were performed to evaluate the postoperative status of vitreoretinal surgery. After exclusions, 50 ultrasounds were reviewed, all performed by the first author using the same equipment. The technique used to image these eyes was contact through the lids in 46 cases, immersion in 3 and both in 1 case. Other techniques such as different patient positioning was used in special cases. Results: Of the 50 patients studied, 30 were males and 20 females. The ages varied between 1 and 82 years, mean of $44.74 \pm 21.09$ years. Of the surgeries performed, 13 were scleral buckles and 37 were pars plana vitrectomies. The time when the examination was requested varied from the $1^{\text {st }}$ to the $48^{\text {th }}$ month after surgery, mean of $6.94 \pm 10.19$ months. The main indications for the study were: impossible fundus examination in 16 cases (32\%), vitreous hemorrhage in 17 cases (34\%) and cataract in 9 cases (18\%). Of the 50 studied eyes, $25(50 \%)$ presented an attached retina and $24(48 \%)$ presented a detached retina. Considering the 37 vitrectomized eyes, $26(52 \%)$ had intraocular silicone oil, 8 had saline solution/aqueous, 1 (2\%) had gas (C3F8), $1(2 \%)$ had gas and residual perfluorocarbon and 1 had perfluorocarbon liquid. Conclusion: B-scan ultrasound enables a convenient, non invasive assessment to evaluate intraocular structures in situations of media opacity after vitreoretinal surgery.

Keywords: Ultrassonography/methods; Vitreous body/ultrassonography; Vitreous body/surgery; Postoperatie period; Evaluation studies 


\section{REFERÊNCIAS}

1. Fisher YL. Diagnostic ophthalmic ultrasonography. In: Tasman W, Jaeger EA, editors. Duane's Foundations of clinical ophthalmology. Revised edition 1997. Philadelphia: Lippincott-Raven; 1997. p.1-10.

2. Kendall CJ. Diagnostic B-scan. In: Kendall C. Ophthalmic echography. Thorofare: Slack; 1990. p.113-44.

3. Lindgren G, Sjodell L, Lindblom B. A prospective study of dense spontaneous vitreous hemorrhage. Am J Ophthalmol 1995;119:458-65.

4. Smith ME, Haik BG, Coleman DJ. Diagnostic ocular ultrasonography. In: Masters B, editor. Noninvasive diagnostic techniques in ophthalmology. New York: Springer-Verlag; 1990. p.47-60.

5. DiBernardo C, Schachat A, Fekrat S. Ophthalmic ultrasound-A diagnostic atlas. New York: Thieme; 1998.

6. Cialdini A, Chedid N, Paranhos F, Ávila M. Ultra-sonografia em patologias vítreo-retinianas. In: Abreu G, editor. Ultra-sonografia ocular: atlas e texto. Rio de Janeiro: Cultura Médica, 2002. p.87-100.

7. Fisher YL, Ciardella AP. Contact B-scan ultrasonography. In: Yanoff M, Duker JS, editors. Ophthalmology. London Philadelphia: Mosby, 1998. p.1-2.

8. Azzolini C, Pierro L, Candino M, Brancato R. Reliability of preoperative ultrasonography evaluation for vitreoretinal surgery. Eur J Ophthalmol 1994; 4:82-90.

9. Câmara AG, Yamane R, Poletti S. Ultra-sonografia. In: Yamane R, editor. Semiologia ocular. Rio de Janeiro: Cultura Médica; 1990. p.229-49.

10. Kumar A, Sharma N, Singh R. Prone position ultrasonography in silicone filled eyes. Acta Ophthalmol Scand 1998;76:496-8.

11. Coleman DJ, Daly SW, Atencio A, Lloyd HO, Silverman RH. Ultrasonic evaluation of the vitreous and retina. Sem Ophthalmol 1998;13:210-8.

12. Hasenfratz G, De La Torre M, Haigis W. Evaluation of eyes harbouring perfluorocarbon liquid with standardized ophthalmic echography. Ger J Ophthalmol 1994;3:19-21. 